\title{
Design, Synthesis and Biological Evaluation of Hydroxy- or Methoxy- Substituted Phenylmethylenethiosemicarbazones as Tyrosinase Inhibitors
}

\author{
Wei Yi, Ri-Hui CaO, Zhi-Yong Chen, Liang Yu, Lin Ma, and Hua-Can SonG* \\ School of Chemistry and Chemical Engineering, Sun Yat-sen University; 135 Xin Gang West Road, Guangzhou 510275, \\ China. Received June 28, 2009; accepted August 10, 2009; published online August 18, 2009
}

\begin{abstract}
A series of hydroxy- or methoxy-substituted phenylmethylenethiosemicarbazones were designed, synthesized and evaluated as mushroom tyrosinase inhibitors. The results demonstrated that most of target compounds had remarkable inhibitory activities on mushroom tyrosinase. Interestingly, compound $2 \mathrm{~h}$ was found to be the most potent tyrosinase inhibitor with $\mathrm{IC}_{50}$ value of $0.18 \mu \mathrm{M}$. The possible interaction mode between compound $2 \mathrm{~h}$ and tyrosinase was proposed. In addition, the 1,1-diphenyl-2-picrylhydrazyl (DPPH) radical scavenging activities of select compounds $\left(\mathrm{IC}_{\mathbf{5 0}}<\mathbf{1 0 . 0} \mu \mathrm{M}\right)$ were also investigated. Compounds $\mathbf{2 d}, \mathbf{2 e}, \mathbf{2} \mathrm{h}, \mathbf{2 \mathrm { i }}$ and $2 \mathrm{l}$ exhibited more potent DPPH radical scavenging activity than well-known antioxidants ascorbic acid ( $\mathrm{Vc})$ and tertiary butyl hydroquinone (TBHQ). These results suggested that such compounds might be utilized for the development of new candidate for treatment of dermatological disorders.
\end{abstract}

Key words phenylmethylenethiosemicarbazone; tyrosinase inhibitor; inhibition mechanism; 1,1-diphenyl-2-picrylhydrazyl radical scavenging activity

Tyrosinase (EC 1.14.18.1; polyphenol oxidase, PPO), a multifunctional copper-containing enzyme, is widely distributed in nature. It catalyzes two distinct reactions of melanin synthesis: the hydroxylation of monophenols and the oxidation of the $o$-phenols. ${ }^{1,2)}$ Tyrosinase is known to be involved in the browning of fruits and vegetables, the formation of brown pigments and the molting process of insects. Therefore, tyrosinase inhibitors have become increasingly important in agriculture, ${ }^{2,3)}$ cosmetic industry ${ }^{4)}$ and medication ${ }^{5)}$ due to decreasing the excessive accumulation of pigmentation resulting from the enzyme action. ${ }^{6-11)}$

Presently, tyrosinase inhibitors have been established as important constituents of cosmetic materials, food preservative and depigmenting agents for hyperpigmentation. ${ }^{12)}$ Many efforts have been spent in the search for effective and safe tyrosinase inhibitors, and a large number of naturally occurring and synthetic tyrosinase inhibitors have already been reported. ${ }^{13-18)}$ However, most of them are not potent enough to put into practical use due to their weak individual activities or safety concerns. Undoubtedly, this is still needed to search and develop novel tyrosinase inhibitors with better activities together with lower side effects.

In the last decades, benzaldehydes, especially its hydroxylated analogues, have been extensively investigated due to their favorable interaction with the hydrophobic protein pocket surrounding the binuclear copper active site of tyrosinase and their lower side effects in vivo studies, such as $\mathrm{N}$ hydroxycinnamoylphenalkyl amides ${ }^{19)}$ aurones,${ }^{10)}$ hydroxysubstituted benzaldoximes, ${ }^{17)}$ chalcones (Fig. 1). ${ }^{3,20)}$ More recently, our groups described the inhibitory effects of 1-(1arylethylidene)thiosemicarbazide and alkylidenethiosemicarbazide derivatives on mushroom tyrosinase (Fig. 1). ${ }^{21,22)}$ The results showed that these compounds exhibited potent inhibitory activity, and the reason was that sulfur atom of thiosemicarbazide moiety was able to chelate the two copper ions in the active site of tyrosinase.

Taking advantage of above information, we speculated that condensation products of hydroxy- or methoxy-substituted benzaldehydes with thiosemicarbazide might exhibit potent tyrosinase inhibitiory activity. Therefore, in continuing our program aimed to search for tyrosinase inhibitors, a series of phenylmethylenethiosemicarbazones were synthesized and their inhibitory effects on the diphenolase activitiy of mushroom tyrosinase were evaluated. In additon, the 1,1-diphenyl2-picrylhydrazyl (DPPH) radical scavenging activities of selected compounds were also investigated. To the best of our knowledge, this is the first time to report the inhibitory effects on the diphenolase activity of mushroom tyrosinase of

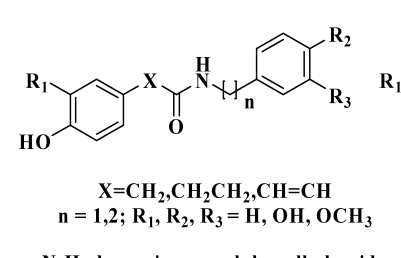

$\mathrm{N}$-Hydroxycinnamoylphenalkylamides

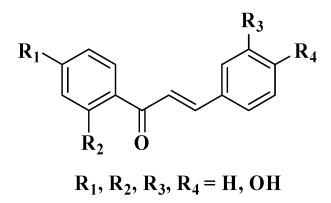

Chalcones
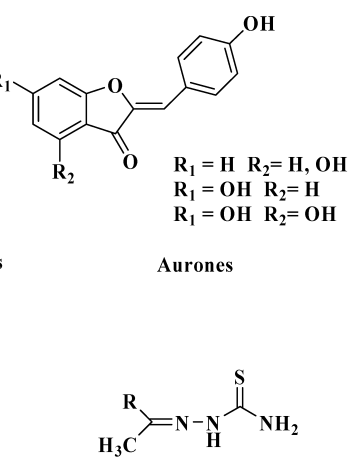

Thiosemicarbazide derivatives
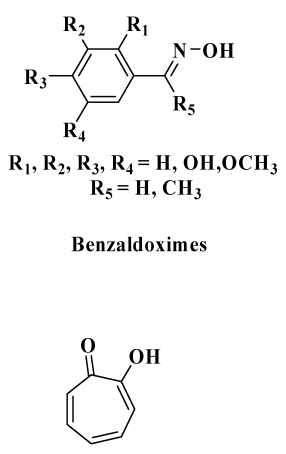

Tropolone

Fig. 1. Chemical Structures of Well-Known Tyrosinase Inhibitors 
phenylmethylenethiosemicarbazones.

\section{Experimental}

Chemistry Melting points ( $\mathrm{mp}$ ) were determined with WRS-1B melting point apparatus and the thermometer was uncorrected. NMR spectra were recorded on Mercury-Plus 300 spectrometers at $25^{\circ} \mathrm{C}$ in deuterochloroform $\left(\mathrm{CDCl}_{3}\right)$ or dimethyl sulfoxide- $d_{6}\left(\mathrm{DMSO}-d_{6}\right)$. All chemical shifts $(\delta)$ are quoted in ppm downfield from TMS and coupling constants $(J)$ are given in Hz. LC-MS spectra were recorded using the LCMS-2010A. All reactions were monitored by TLC (Merck Kieselgel $60 \mathrm{~F}_{254}$ ) and the spots were visualized under UV light. Elemental analyses were performed on a Vario EL instrument and were within $\pm 0.4 \%$ of the theoretical values. The benzylaldehydes, ketones, thiosemicarbazide and 4-methoxycinnamic acid were purchased from Darui Chemical Co. (ShangHai, China). Mushroom tyrosinase (specific activity of the enzyme is $6680 \mathrm{U} / \mathrm{mg}$ ) and L-3,4-dihydroxyphenylalanine (L-DOPA) were purchased from Sigma Chemical Co. All commercially available reagents and solvents were used without further purification.

Synthesis To the solution of appropriate aldehydes $(10 \mathrm{mmol})$ in anhydrous ethanol $(10 \mathrm{ml})$, thiosemicarbazide $(10 \mathrm{mmol})$ and acetic acid $(0.5 \mathrm{ml})$ were added. The reaction mixture was refluxed for $24 \mathrm{~h}$ and cooled to room temperature. The precipitate solid was filtered, washed with ether, and purified by recrystallization from $95 \%$ ethanol to afford compounds $\mathbf{2 a}-\mathbf{r}$.

2-(Phenylmethylene)-thiosemicarbazone (2a): Yield 76\%, mp $153-154{ }^{\circ} \mathrm{C}$. ${ }^{1} \mathrm{H}-\mathrm{NMR}\left(\mathrm{DMSO}-d_{6}, 300 \mathrm{MHz}\right) \delta: 11.40(1 \mathrm{H}, \mathrm{s}, \mathrm{NH}), 8.17\left(1 \mathrm{H}\right.$, br s, $\left.\mathrm{NH}_{2}\right)$, $8.03(\mathrm{~s}, 1 \mathrm{H},-\mathrm{HC}=\mathrm{N}-), 7.96\left(1 \mathrm{H}\right.$, br s, $\left.\mathrm{NH}_{2}\right), 7.77(2 \mathrm{H}, \mathrm{m}, \mathrm{phH}), 7.38(3 \mathrm{H}$, m, phH). MS (ESI): $m / z(100 \%)=180(\mathrm{M}+1)$. Anal. Calcd for $\mathrm{C}_{8} \mathrm{H}_{9} \mathrm{~N}_{3} \mathrm{~S}: \mathrm{C}$, 53.61; H, 5.06; N, 23.44. Found: C, 53.25; H, 5.24; N, 23.21.

2-[(2-Hydroxyphenyl)methylene]-thiosemicarbazone (2b): Yield 66\%, mp $219-220{ }^{\circ} \mathrm{C} .{ }^{1} \mathrm{H}-\mathrm{NMR}\left(\mathrm{DMSO}-d_{6}, 300 \mathrm{MHz}\right) \delta: 11.33(1 \mathrm{H}, \mathrm{s}, \mathrm{NH}), 9.84$ $(1 \mathrm{H}, \mathrm{s}, \mathrm{OH}), 8.34(\mathrm{~s}, 1 \mathrm{H},-\mathrm{HC}=\mathrm{N}-), 8.07\left(1 \mathrm{H}\right.$, br s, $\left.\mathrm{NH}_{2}\right), 7.90(1 \mathrm{H}$, br s, $\left.\mathrm{NH}_{2}\right), 7.87(1 \mathrm{H}, \mathrm{d}, J=7.5 \mathrm{~Hz}, \mathrm{phH}), 7.18(1 \mathrm{H}$, 't', $J=8.1 \mathrm{~Hz}, \mathrm{phH}), 6.83(1 \mathrm{H}$, d, $J=7.6 \mathrm{~Hz}, \mathrm{phH}), 6.79(1 \mathrm{H}$, 't', $J=7.6 \mathrm{~Hz}, \mathrm{phH}) . \mathrm{MS}$ (ESI): $\mathrm{m} / \mathrm{z}$ $(100 \%)=196(\mathrm{M}+1)$. Anal. Calcd for $\mathrm{C}_{8} \mathrm{H}_{9} \mathrm{~N}_{3} \mathrm{OS}: \mathrm{C}, 49.21 ; \mathrm{H}, 4.65 ; \mathrm{N}$, 21.52. Found: C, 49.04; H, 4.64; N, 21.61.

2-[(3-Hydroxyphenyl)methylene]-thiosemicarbazone (2c): Yield 83\%, mp $168-170^{\circ} \mathrm{C} .{ }^{1} \mathrm{H}-\mathrm{NMR}$ (DMSO-d, $\left.300 \mathrm{MHz}\right) \delta: 11.34(1 \mathrm{H}, \mathrm{s}, \mathrm{NH}), 9.50$ $(1 \mathrm{H}, \mathrm{s}, \mathrm{OH}), 8.15\left(1 \mathrm{H}\right.$, br s, $\left.\mathrm{NH}_{2}\right), 7.95(\mathrm{~s}, 1 \mathrm{H},-\mathrm{HC}=\mathrm{N}-), 7.86(1 \mathrm{H}$, br s, $\left.\mathrm{NH}_{2}\right), 7.19(1 \mathrm{H}, \mathrm{s}, \mathrm{phH}), 7.17(1 \mathrm{H}, \mathrm{d}, J=7.6 \mathrm{~Hz}, \mathrm{phH}), 7.13(1 \mathrm{H}, \mathrm{d}, J=$ $7.3 \mathrm{~Hz}, \mathrm{phH}), 6.79(1 \mathrm{H}, \mathrm{m}, \mathrm{phH})$. MS (ESI): $m / z(100 \%)=196(\mathrm{M}+1)$. Anal. Calcd for $\mathrm{C}_{8} \mathrm{H}_{9} \mathrm{~N}_{3} \mathrm{OS}$ : C, 49.21; H, 4.65; N, 21.52. Found: C, 49.15; H, 4.82; $\mathrm{N}, 21.28$.

2-[(4-Hydroxyphenyl)methylene]-thiosemicarbazone (2d): Yield 52\%, mp $218-219^{\circ} \mathrm{C} .{ }^{1} \mathrm{H}-\mathrm{NMR}$ (DMSO- $\left.d_{6}, 300 \mathrm{MHz}\right) \delta: 11.21(1 \mathrm{H}, \mathrm{s}, \mathrm{NH}), 9.83$ $(1 \mathrm{H}, \mathrm{s}, \mathrm{OH}), 8.03\left(1 \mathrm{H}\right.$, br s, $\left.\mathrm{NH}_{2}\right), 7.93(\mathrm{~s}, 1 \mathrm{H},-\mathrm{HC}=\mathrm{N}-), 7.80(1 \mathrm{H}$, br s, $\left.\mathrm{NH}_{2}\right), 7.59(2 \mathrm{H}, \mathrm{d}, J=8.3 \mathrm{~Hz}, \mathrm{phH}), 6.75(2 \mathrm{H}, \mathrm{d}, J=8.3 \mathrm{~Hz}, \mathrm{phH}) . \mathrm{MS}(\mathrm{ESI})$ $m / z(100 \%)=196(\mathrm{M}+1)$. Anal. Calcd for $\mathrm{C}_{8} \mathrm{H}_{9} \mathrm{~N}_{3} \mathrm{OS}: \mathrm{C}, 49.21 ; \mathrm{H}, 4.65 ; \mathrm{N}$, 21.52. Found: C, 49.49; H, 4.66; N, 21.38.

2-[(4-Methoxyphenyl)methylene]-thiosemicarbazone (2e): Yield 75\%, mp $166-168^{\circ} \mathrm{C} .{ }^{1} \mathrm{H}-\mathrm{NMR}$ (DMSO- $\left.d_{6}, 300 \mathrm{MHz}\right) \delta: 11.28(1 \mathrm{H}, \mathrm{s}, \mathrm{NH}), 8.08$ $\left(1 \mathrm{H}\right.$, brs, $\left.\mathrm{NH}_{2}\right), 7.97(\mathrm{~s}, 1 \mathrm{H},-\mathrm{HC}=\mathrm{N}-), 7.88\left(1 \mathrm{H}\right.$, br s, $\left.\mathrm{NH}_{2}\right), 7.71(2 \mathrm{H}, \mathrm{d}$, $J=8.7 \mathrm{~Hz}, \mathrm{phH}), 6.94(2 \mathrm{H}, \mathrm{d}, J=8.7 \mathrm{~Hz}, \mathrm{phH}), 3.78\left(3 \mathrm{H}, \mathrm{s}, \mathrm{CH}_{3}\right) .{ }^{13} \mathrm{C}-\mathrm{NMR}$ $\left(75 \mathrm{MHz}, \mathrm{DMSO}-d_{6}\right) \delta: 178.1,161.3,142.9,129.5,127.4,114.8,56.1 . \mathrm{MS}$ (ESI): $m / z(100 \%)=210(\mathrm{M}+1)$. Anal. Calcd for $\mathrm{C}_{9} \mathrm{H}_{11} \mathrm{~N}_{3} \mathrm{OS}: \mathrm{C}, 51.65 ; \mathrm{H}$, 5.30; N, 20.08. Found: C, 51.78; H, 5.52; N, 19.93 .

2-[(4-Bromophenyl)methylene]-thiosemicarbazone (2f): Yield 80\%, mp $203-204{ }^{\circ} \mathrm{C}$. ${ }^{1} \mathrm{H}-\mathrm{NMR}$ (DMSO-d, $\left.300 \mathrm{MHz}\right) \delta: 11.45(1 \mathrm{H}, \mathrm{s}, \mathrm{NH}), 8.21$ $\left(1 \mathrm{H}\right.$, brs, $\left.\mathrm{NH}_{2}\right), 8.04\left(1 \mathrm{H}\right.$, br s, $\left.\mathrm{NH}_{2}\right), 7.98(\mathrm{~s}, 1 \mathrm{H},-\mathrm{HC}=\mathrm{N}-), 7.73(2 \mathrm{H}, \mathrm{d}$, $J=8.1 \mathrm{~Hz}, \mathrm{phH}), 7.55(2 \mathrm{H}, \mathrm{d}, J=8.1 \mathrm{~Hz}, \mathrm{phH}) .{ }^{13} \mathrm{C}-\mathrm{NMR}(75 \mathrm{MHz}, \mathrm{DMSO}-$ $\left.d_{6}\right) \delta: 178.6,141.5,134.1,132.2,129.8,123.7 . \mathrm{MS}(\mathrm{ESI}): m / z(100 \%)=258$ $(\mathrm{M}+1)$. Anal. Calcd for $\mathrm{C}_{8} \mathrm{H}_{8} \mathrm{BrN}_{3} \mathrm{~S}: \mathrm{C}, 37.22 ; \mathrm{H}, 3.12 ; \mathrm{N}, 16.28$. Found: C, $37.19 ; \mathrm{H}, 3.26 ; \mathrm{N}, 16.42$.

2-[(2-Hydroxy-4-bromophenyl)methylene]thiosemicarbazone (2g): Yield $79 \%, \mathrm{mp} 230-231^{\circ} \mathrm{C} .{ }^{1} \mathrm{H}-\mathrm{NMR}$ (DMSO-d, $\left.300 \mathrm{MHz}\right) \delta: 11.38(1 \mathrm{H}, \mathrm{s}$, $\mathrm{NH}), 10.19(1 \mathrm{H}, \mathrm{s}, \mathrm{OH}), 8.27(1 \mathrm{H}, \mathrm{s},-\mathrm{HC}=\mathrm{N}-), 8.17(1 \mathrm{H}, \mathrm{s}, \mathrm{phH}), 8.13$ $\left(2 \mathrm{H}\right.$, br s, $\left.\mathrm{NH}_{2}\right), 7.30(1 \mathrm{H}, \mathrm{dd}, J=2.1,8.5 \mathrm{~Hz}, \mathrm{phH}), 6.80(1 \mathrm{H}, \mathrm{d}, J=8.8 \mathrm{~Hz}$, phH). MS (ESI): $m / z(100 \%)=274(\mathrm{M}+1)$. Anal. Calcd for $\mathrm{C}_{8} \mathrm{H}_{8} \mathrm{BrN}_{3} \mathrm{OS}: \mathrm{C}$, 35.05; H, 2.94; N, 15.33. Found: C, 34.96; H, 2.88; N, 15.56.

2-[(2,4-Dihydroxyphenyl)methylene]-thiosemicarbazone (2h): Yield 68\%, mp $228-230{ }^{\circ} \mathrm{C} .{ }^{1} \mathrm{H}-\mathrm{NMR}$ (DMSO- $\left.d_{6}, 300 \mathrm{MHz}\right) \delta: 11.14(1 \mathrm{H}, \mathrm{s}, \mathrm{NH}$ ), $9.72(2 \mathrm{H}, \mathrm{s}, \mathrm{OH}), 8.22(\mathrm{~s}, 1 \mathrm{H},-\mathrm{HC}=\mathrm{N}-), 7.93\left(1 \mathrm{H}\right.$, br s, $\left.\mathrm{NH}_{2}\right), 7.72(1 \mathrm{H}$, br s, $\left.\mathrm{NH}_{2}\right), 7.65(1 \mathrm{H}, \mathrm{d}, J=8.5 \mathrm{~Hz}, \mathrm{phH}), 6.27(1 \mathrm{H}, \mathrm{s}, \mathrm{phH}), 6.24(1 \mathrm{H}, \mathrm{d}$, $J=8.8 \mathrm{~Hz}, \mathrm{phH})$. MS (ESI): $m / z(100 \%)=212(\mathrm{M}+1)$. Anal. Calcd for $\mathrm{C}_{8} \mathrm{H}_{9} \mathrm{~N}_{3} \mathrm{O}_{2} \mathrm{~S}$ : C, 45.49; H, 4.29; N, 19.89. Found: C, 45.60; H, 4.22; N, 20.22 .
2-[(2,5-Dihydroxyphenyl)methylene]-thiosemicarbazone (2i): Yield 61\%, mp $237-239^{\circ} \mathrm{C} .{ }^{1} \mathrm{H}-\mathrm{NMR}$ (DMSO- $\left.d_{6}, 300 \mathrm{MHz}\right) \delta: 11.30(1 \mathrm{H}, \mathrm{s}, \mathrm{NH})$, $9.15(1 \mathrm{H}, \mathrm{s}, \mathrm{OH}), 8.76(1 \mathrm{H}, \mathrm{s}, \mathrm{OH}), 8.27(\mathrm{~s}, 1 \mathrm{H},-\mathrm{HC}=\mathrm{N}-), 8.05(1 \mathrm{H}, \mathrm{br}$, $\left.\mathrm{NH}_{2}\right), 7.76\left(1 \mathrm{H}\right.$, br s, $\left.\mathrm{NH}_{2}\right), 7.20(1 \mathrm{H}, \mathrm{s}, \mathrm{phH}), 6.66(1 \mathrm{H}, \mathrm{d}, J=7.8 \mathrm{~Hz}, \mathrm{phH})$, $6.73(1 \mathrm{H}, \mathrm{d}, J=8.1 \mathrm{~Hz}, \mathrm{phH})$. MS (ESI): $m / z(100 \%)=212(\mathrm{M}+1)$. Anal. Calcd for $\mathrm{C}_{8} \mathrm{H}_{9} \mathrm{~N}_{3} \mathrm{O}_{2} \mathrm{~S}$ : C, 45.49; H, 4.29; N, 19.89. Found: C, 45.31; H, $4.41 ; \mathrm{N}, 20.08$.

2-[(3,5-Dihydroxyphenyl)methylene]-thiosemicarbazone (2j): Yield 62\%, $\mathrm{mp}>250{ }^{\circ} \mathrm{C} .{ }^{1} \mathrm{H}-\mathrm{NMR}\left(\mathrm{DMSO}-d_{6}, 300 \mathrm{MHz}\right) \delta: 11.27(1 \mathrm{H}, \mathrm{s}, \mathrm{NH}), 9.34$ $(2 \mathrm{H}, \mathrm{s}, \mathrm{OH}), 8.11\left(1 \mathrm{H}\right.$, br s, $\left.\mathrm{NH}_{2}\right), 7.83(\mathrm{~s}, 1 \mathrm{H},-\mathrm{HC}=\mathrm{N}-), 7.76(1 \mathrm{H}$, br s, $\left.\mathrm{NH}_{2}\right), 6.56(2 \mathrm{H}, \mathrm{d}, J=2.1 \mathrm{~Hz}, \mathrm{phH}), 6.25(1 \mathrm{H}$, 't', $J=2.2 \mathrm{~Hz}, \mathrm{phH}) . \mathrm{MS}$ (ESI): $m / z(100 \%)=212(\mathrm{M}+1)$. Anal. Calcd for $\mathrm{C}_{8} \mathrm{H}_{9} \mathrm{~N}_{3} \mathrm{O}_{2} \mathrm{~S}: \mathrm{C}, 45.49 ; \mathrm{H}$, 4.29; N, 19.89. Found: C, 45.45; H, 4.40; N, 19.98

2-[(3,4-Dihydroxyphenyl)methylene]-thiosemicarbazone (2k): Yield 73\%, mp $234-235^{\circ} \mathrm{C} .{ }^{1} \mathrm{H}-\mathrm{NMR}$ (DMSO-d, $\left.300 \mathrm{MHz}\right) \delta: 11.17(1 \mathrm{H}, \mathrm{s}, \mathrm{NH})$ $9.45(1 \mathrm{H}, \mathrm{s}, \mathrm{OH}), 8.97(1 \mathrm{H}, \mathrm{s}, \mathrm{OH}), 8.01\left(1 \mathrm{H}, \mathrm{br}, \mathrm{NH}_{2}\right), 7.86(\mathrm{~s}, 1 \mathrm{H},-\mathrm{HC}=$ $\mathrm{N}-), 7.70\left(1 \mathrm{H}\right.$, brs, $\left.\mathrm{NH}_{2}\right), 7.15(1 \mathrm{H}, \mathrm{s}, \mathrm{phH}), 7.01(1 \mathrm{H}, \mathrm{d}, J=8.1 \mathrm{~Hz}, \mathrm{phH})$, $6.73(1 \mathrm{H}, \mathrm{d}, J=8.1 \mathrm{~Hz}, \mathrm{phH}) .{ }^{13} \mathrm{C}-\mathrm{NMR}\left(75 \mathrm{MHz}, \mathrm{DMSO}-d_{6}\right) \delta: 177.9$, $148.3,146.1,143.9,126.2,120.8,116.2,114.5$. MS (ESI): $m / z(100 \%)=212$ $(\mathrm{M}+1)$. Anal. Calcd for $\mathrm{C}_{8} \mathrm{H}_{9} \mathrm{~N}_{3} \mathrm{O}_{2} \mathrm{~S}: \mathrm{C}, 45.49 ; \mathrm{H}, 4.29 ; \mathrm{N}, 19.89$. Found: C, 45.25; H, 4.43; N, 20.25.

2-[(3-Methoxy-4-hydroxyphenyl)methylene]-thiosemicarbazone (2l): Yield $85 \%, \mathrm{mp} 194-195^{\circ} \mathrm{C} .{ }^{1} \mathrm{H}-\mathrm{NMR}$ (DMSO- $\left.d_{6}, 300 \mathrm{MHz}\right) \delta: 11.21(1 \mathrm{H}$, s, NH), $9.41(1 \mathrm{H}, \mathrm{s}, \mathrm{OH}), 8.08\left(1 \mathrm{H}\right.$, br s, $\left.\mathrm{NH}_{2}\right), 7.92\left(1 \mathrm{H}\right.$, br s, $\left.\mathrm{NH}_{2}\right), 7.90(\mathrm{~s}$ $1 \mathrm{H},-\mathrm{HC}=\mathrm{N}-), 7.44(1 \mathrm{H}, \mathrm{s}, \mathrm{phH}), 7.01(1 \mathrm{H}, \mathrm{d}, J=8.3 \mathrm{~Hz}, \mathrm{phH}), 6.75(1 \mathrm{H}, \mathrm{d}$, $J=8.2 \mathrm{~Hz}, \mathrm{phH}), 3.81\left(3 \mathrm{H}, \mathrm{s}, \mathrm{CH}_{3}\right)$. MS (ESI): $m / z(100 \%)=226(\mathrm{M}+1)$ Anal. Calcd for $\mathrm{C}_{9} \mathrm{H}_{11} \mathrm{~N}_{3} \mathrm{O}_{2} \mathrm{~S}$ : C, 47.99; H, 4.92; N, 18.65. Found: C, 48.13; $\mathrm{H}, 4.88 ; \mathrm{N}, 18.83$

2-[(3-Hydroxy-4-methoxyphenyl)methylene]-thiosemicarbazone $\quad(\mathbf{2 m})$ : Yield $88 \%, \operatorname{mp~} 176-177^{\circ} \mathrm{C} .{ }^{1} \mathrm{H}-\mathrm{NMR}$ (DMSO- $\left.d_{6}, 300 \mathrm{MHz}\right) \delta: 11.23(1 \mathrm{H}$, s, NH), $9.02(1 \mathrm{H}, \mathrm{s}, \mathrm{OH}), 8.05\left(1 \mathrm{H}\right.$, br s, $\left.\mathrm{NH}_{2}\right), 7.89(\mathrm{~s}, 1 \mathrm{H},-\mathrm{HC}=\mathrm{N}-), 7.78$ $\left(1 \mathrm{H}\right.$, br s, $\left.\mathrm{NH}_{2}\right), 7.23(1 \mathrm{H}, \mathrm{s}, \mathrm{phH}), 7.08(1 \mathrm{H}, \mathrm{d}, J=7.8 \mathrm{~Hz}, \mathrm{phH}), 6.90(1 \mathrm{H}, \mathrm{d}$, $J=7.8 \mathrm{~Hz}, \mathrm{phH}), 3.78\left(3 \mathrm{H}, \mathrm{s}, \mathrm{CH}_{3}\right)$. MS (ESI): $m / z(100 \%)=226(\mathrm{M}+1)$. Anal. Calcd for $\mathrm{C}_{9} \mathrm{H}_{11} \mathrm{~N}_{3} \mathrm{O}_{2} \mathrm{~S}$ : C, 47.99; H, 4.92; N, 18.65. Found: C, 48.15; $\mathrm{H}, 4.81 ; \mathrm{N}, 18.91$.

2-[(2,5-Dimethoxyphenyl)methylene]-thiosemicarbazone (2n): Yield 82\%, mp $221-222{ }^{\circ} \mathrm{C} .{ }^{1} \mathrm{H}-\mathrm{NMR}$ (DMSO- $\left.d_{6}, 300 \mathrm{MHz}\right) \delta: 11.36(1 \mathrm{H}, \mathrm{s}, \mathrm{NH}), 8.35$ $(\mathrm{s}, 1 \mathrm{H},-\mathrm{HC}=\mathrm{N}-), 8.14\left(1 \mathrm{H}\right.$, br s, $\left.\mathrm{NH}_{2}\right), 8.04\left(1 \mathrm{H}\right.$, brs, $\left.\mathrm{NH}_{2}\right), 7.61(1 \mathrm{H}, \mathrm{d}$, $J=2.8 \mathrm{~Hz}, \mathrm{phH}), 6.93(2 \mathrm{H}, \mathrm{m}, \mathrm{phH}), 3.75\left(3 \mathrm{H}, \mathrm{s}, \mathrm{CH}_{3}\right), 3.73\left(3 \mathrm{H}, \mathrm{s}, \mathrm{CH}_{3}\right)$. ${ }^{13} \mathrm{C}-\mathrm{NMR}\left(75 \mathrm{MHz}\right.$, DMSO- $\left.d_{6}\right) \delta$ : 178.4, 153.0, 152.9, 138.5, 123.5, 118.1, 113.8, 110.7, 57.0, 56.4. MS (ESI): $m / z(100 \%)=240(\mathrm{M}+1)$. Anal. Calcd for $\mathrm{C}_{10} \mathrm{H}_{13} \mathrm{~N}_{3} \mathrm{O}_{2} \mathrm{~S}$ : C, 50.19; H, 5.48; N, 17.56. Found: C, 50.41; H, 5.32; N, 17.79 .

2-[(2,3,4-Trihydroxy)methylene]-thiosemicarbazone (2o): Yield 78\%, mp $248-249^{\circ} \mathrm{C}$. ${ }^{1} \mathrm{H}-\mathrm{NMR}$ (DMSO- $\left.d_{6}, 300 \mathrm{MHz}\right) \delta: 11.16(1 \mathrm{H}, \mathrm{s}, \mathrm{NH}), 9.48$ $(1 \mathrm{H}$, brs, $\mathrm{OH}), 8.94(1 \mathrm{H}$, brs, $\mathrm{OH}), 8.40\left(1 \mathrm{H}\right.$, brs, $\left.\mathrm{NH}_{2}\right), 8.20(\mathrm{~s}, 1 \mathrm{H}$, $-\mathrm{HC}=\mathrm{N}-), 7.93(1 \mathrm{H}$, br s, OH $), 7.72\left(1 \mathrm{H}\right.$, br s, $\left.\mathrm{NH}_{2}\right), 7.11(1 \mathrm{H}, \mathrm{d}, J=8.4 \mathrm{~Hz}$, phH), $6.32(1 \mathrm{H}, \mathrm{d}, J=8.4 \mathrm{~Hz}, \mathrm{phH})$. MS (ESI): $m / z(100 \%)=228(\mathrm{M}+1)$ Anal. Calcd for $\mathrm{C}_{8} \mathrm{H}_{9} \mathrm{~N}_{3} \mathrm{O}_{3} \mathrm{~S}$ : C, 42.28; H, 3.99; N, 18.49. Found: C, 42.33; $\mathrm{H}, 4.07 ; \mathrm{N}, 18.31$.

2-[(3,4,5-Trihydroxy)methylene]-thiosemicarbazone (2p): Yield 85\%, mp $237-239^{\circ} \mathrm{C} .{ }^{1} \mathrm{H}-\mathrm{NMR}$ (DMSO-d, $\left.300 \mathrm{MHz}\right) \delta: 11.13(1 \mathrm{H}, \mathrm{s}, \mathrm{NH}), 8.96$ $(2 \mathrm{H}$, brs, $\mathrm{OH}), 8.62(1 \mathrm{H}$, brs, OH $), 8.01\left(1 \mathrm{H}\right.$, brs, $\left.\mathrm{NH}_{2}\right), 7.78(\mathrm{~s}, 1 \mathrm{H}$, $-\mathrm{HC}=\mathrm{N}-), 7.59\left(1 \mathrm{H}\right.$, br s, $\left.\mathrm{NH}_{2}\right), 6.64(2 \mathrm{H}, \mathrm{s}, \mathrm{phH}) .{ }^{13} \mathrm{C}-\mathrm{NMR}(75 \mathrm{MHz}$, DMSO- $\left.d_{6}\right) \delta: 177.9,146.7,144.4,136.4,125.0,107.3$. MS (ESI): $m / z$ $(100 \%)=228(\mathrm{M}+1)$. Anal. Calcd for $\mathrm{C}_{8} \mathrm{H}_{9} \mathrm{~N}_{3} \mathrm{O}_{3} \mathrm{~S}: \mathrm{C}, 42.28 ; \mathrm{H}, 3.99 ; \mathrm{N}$, 18.49. Found: C, 42.19; H, 4.07; N, 18.65 .

2-[(3,4,5-Trimethoxy)methylene]-thiosemicarbazone (2q): Yield $87 \%, \mathrm{mp}$ $211-212{ }^{\circ} \mathrm{C}$. ${ }^{1} \mathrm{H}-\mathrm{NMR}$ (DMSO- $\left.d_{6}, 300 \mathrm{MHz}\right) \delta$ : $11.40(1 \mathrm{H}, \mathrm{s}, \mathrm{NH}), 8.21$ $\left(1 \mathrm{H}\right.$, br s, $\left.\mathrm{NH}_{2}\right), 8.07\left(1 \mathrm{H}\right.$, brs, $\left.\mathrm{NH}_{2}\right), 7.93(\mathrm{~s}, 1 \mathrm{H},-\mathrm{HC}=\mathrm{N}-), 7.06(2 \mathrm{H}, \mathrm{s}$, $\mathrm{phH}), 3.81\left(6 \mathrm{H}, \mathrm{s}, 2 \times \mathrm{CH}_{3}\right), 3.66\left(3 \mathrm{H}, \mathrm{s}, \mathrm{CH}_{3}\right) .{ }^{13} \mathrm{C}-\mathrm{NMR}(75 \mathrm{MHz}, \mathrm{DMSO}-$ $\left.d_{6}\right) \delta: 178.3,153.7,142.8,139.6,130.3,105.4,60.8,56.8$. MS (ESI): $m / z$ $(100 \%)=270(\mathrm{M}+1)$. Anal. Calcd for $\mathrm{C}_{11} \mathrm{H}_{15} \mathrm{~N}_{3} \mathrm{O}_{3} \mathrm{~S}: \mathrm{C}, 49.06 ; \mathrm{H}, 5.61 ; \mathrm{N}$, 15.60. Found: C, 48.99; H, 5.65; N, 15.87 .

2-(2-Furanylmethylene)-thiosemicarbazone (2r): Yield 77\%, mp 143$145^{\circ} \mathrm{C} .{ }^{1} \mathrm{H}-\mathrm{NMR}$ (DMSO- $\left.d_{6}, 300 \mathrm{MHz}\right) \delta: 11.39(1 \mathrm{H}, \mathrm{s}, \mathrm{NH}), 8.18(1 \mathrm{H}$, br s, $\left.\mathrm{NH}_{2}\right), 7.94(\mathrm{~s}, 1 \mathrm{H},-\mathrm{HC}=\mathrm{N}-), 7.78(1 \mathrm{H}, \mathrm{d}, J=1.8 \mathrm{~Hz}$, furylH $), 7.60$ $\left(1 \mathrm{H}\right.$, br s, $\left.\mathrm{NH}_{2}\right), 6.94(1 \mathrm{H}, \mathrm{d}, J=3.4 \mathrm{~Hz}$, furylH $), 6.59(1 \mathrm{H}, \mathrm{m}$, furylH). MS (ESI): $m / z(100 \%)=170(\mathrm{M}+1)$. Anal. Calcd for $\mathrm{C}_{6} \mathrm{H}_{7} \mathrm{~N}_{3} \mathrm{OS}: \mathrm{C}, 42.59 ; \mathrm{H}$, 4.17; N, 24.83. Found: C, 42.86; H, 4.27; N, 24.42 .

Tyrosinase Inhibition Assay Tyrosinase inhibition assays were performed according to the developed method described earlier by Hearing. ${ }^{23)}$ Briefly, all the synthesized compounds were screened for the $o$-diphenolase inhibitory activity of tyrosinase using L-DOPA as substrate. All the active in- 
hibitors from the preliminary screening were subjected to $\mathrm{IC}_{50}$ studies. All the synthesized compounds were dissolved in DMSO to a concentration of $2.0 \%$. Phosphate buffer $\mathrm{pH} 6.8$ was used to dilute the DMSO stock solution of test compound. Thirty units of mushroom tyrosinase $(0.2 \mu \mathrm{g} / \mathrm{ml})$ was first pre-incubated with the compounds, in $50 \mathrm{~mm}$ phosphate buffer $(\mathrm{pH} 6.8)$, for $10 \mathrm{~min}$ at $25^{\circ} \mathrm{C}$. Then the L-DOPA $(0.5 \mathrm{~mm})$ was added to the reaction mixture and the enzyme reaction was monitored by measuring the change in absorbance at $475 \mathrm{~nm}$ of the DOPAchrome for $1 \mathrm{~min}$. $\mathrm{IC}_{50}$ value, a concentration giving $50 \%$ inhibition of tyrosinase activity, was determined by interpolation of the dose-response curves. Here, 4-methoxycinnamic acid, 4-hydroxybenzaldehyde and tropolone were used as the reference inhibitors.

DPPH Radical Scavenging Activity The DPPH radical scavenging activity of all the synthesized compounds was analyzed according to the modified procedure described by Iwai et $_{\text {al. }}{ }^{24)}$ Compounds in DMSO solution (20 $\mu \mathrm{l})$ were added to a mixture of $100 \mathrm{~mm}$ acetate buffer $(\mathrm{pH} 5.5,630 \mu \mathrm{l})$ and $0.3 \mathrm{~mm}$ DPPH in ethanol $(350 \mu \mathrm{l})$ in a test tube and left to stand at room temperature in the dark for $30 \mathrm{~min}$. The absorbances of the resulting solutions were measured at $517 \mathrm{~nm}$. Ascorbic acid (Vc) and tertiary butyl hydroquinone (TBHQ) were used as the reference, and the negative control was prepared without the compounds. The activity was expressed as the concentration of sample necessary to give a $50 \%$ reduction in the sample absorbance $\left(\mathrm{EC}_{50}\right)$.

\section{Results and Discussion}

Synthetic Chemistry The synthesis of compounds $\mathbf{2 a}$ r was summarized in Chart 1 , and the chemical structure of compounds $\mathbf{2} \mathbf{a}-\mathbf{q}$ was given in Table 1 . The condensation of the corresponding benzylaldehydes with thiosemicarbazide could be carried out easily in anhydrous alcohol using acetic acid as catalyst to provide phenylmethylenethiosemicarbazones $\mathbf{2 a}-\mathbf{q}$ and its analogue $\mathbf{2 r}$ in good yield. The chemical structures of all the synthesized compounds were characterized by elemental analysis $(\mathrm{C}, \mathrm{H}, \mathrm{N}), \mathrm{MS},{ }^{1} \mathrm{H}-\mathrm{NMR},{ }^{13} \mathrm{C}-\mathrm{NMR}$ spectra.

Effects on Tyrosinase Activity All the synthesized compounds were subjected to tyrosinase inhibition assay with L-DOPA as substrate, according to the developed method described by Hearing. ${ }^{23)}$ The $\mathrm{IC}_{50}$ values of all compounds investigated were summarized in Table 2. As shown in Table 2, all synthesized compounds (except compound 2o) displayed more potent tyrosinase inhibitory activities than the reference inhibitors, 4-hydroxybenzaldehyde and 4-methoxycinnamic acid. Particularly, compounds $2 \mathbf{b}\left(\mathrm{IC}_{50}=0.38 \mu \mathrm{M}\right), \mathbf{2 f}\left(\mathrm{IC}_{50}=\right.$ $0.28 \mu \mathrm{M}), 2 \mathrm{~g}\left(\mathrm{IC}_{50}=0.33 \mu \mathrm{M}\right)$, and $2 \mathrm{~h}\left(\mathrm{IC}_{50}=0.18 \mu \mathrm{M}\right)$ were found to be the most active compounds of this series, even better than the reference inhibitor tropolone $\left(\mathrm{IC}_{50}=0.42 \mu \mathrm{M}\right.$, Fig. 1). Meanwhile, the $\mathrm{IC}_{50}$ values of compounds $\mathbf{2 d}$ and $\mathbf{2 r}$ were close to that of tropolone. Therefore, these synthesized compounds are worth investigating further.

Of all phenylmethylenethiosemicarbazones $\mathbf{2} \mathbf{a}-\mathbf{q}$, compound $\mathbf{2 a}\left(\mathrm{IC}_{50}=1.93 \mu \mathrm{M}\right)$ having no substituent on phenyl ring exhibited excellent inhibitory effect on mushroom tyrosinase. Introduction of hydroxyl groups onto position-2 and 4 of phenyl ring to afford compounds $2 \mathbf{b}\left(\mathrm{IC}_{50}=0.38 \mu \mathrm{M}\right)$ and $\mathbf{2 d}\left(\mathrm{IC}_{50}=0.41 \mu \mathrm{M}\right)$, respectively, which displayed a significant increase in tyrosinase inhibition potency. Compared to $\mathbf{2 b}$ and $\mathbf{2 d}$, compound $\mathbf{2 c}\left(\mathrm{IC}_{50}=3.90 \mu \mathrm{M}\right)$ bearing a hydroxyl substituent at position-3 of phenyl ring showed a decline in activity. These results suggested that the introduction of hydroxyl group on the phenyl ring obviously affected the tyrosinase inhibitory activity, ${ }^{27)}$ and in the present investigation, the 2- and 4-hydroxyl groups facilitated their inhibitory activities while the 3-hydroxyl substituent was unfavorable.

When the hydroxyl group of compound $\mathbf{2 d}\left(\mathrm{IC}_{50}=0.41\right.$
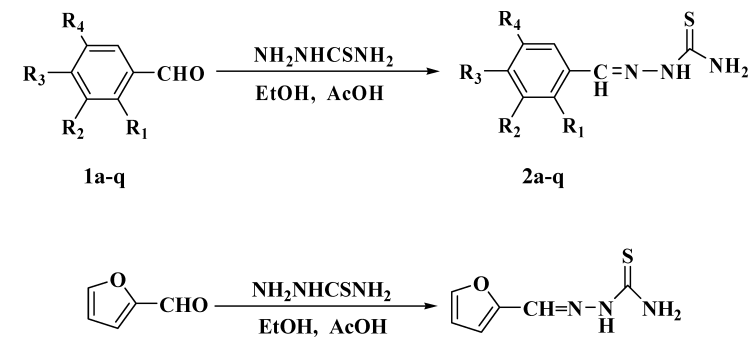

1r

$2 \mathbf{r}$

Chart 1. Synthesis of Phenylmethylenethiosemicarbazones $\mathbf{2} \mathbf{a}-\mathbf{q}$ and its Analogue 2r

Table 1. Chemical Structure of Phenylmethylenethiosemicarbazones $\mathbf{2 a}-\mathbf{q}$<smiles>[R3]c1cc(C=NNC(N)=S)c([R1])c([R3])c1[R3]</smiles>

\begin{tabular}{lllll}
\hline \hline Compound & $\mathrm{R}_{1}$ & $\mathrm{R}_{2}$ & $\mathrm{R}_{3}$ & $\mathrm{R}_{4}$ \\
\hline $\mathbf{2 a}$ & $\mathrm{H}$ & $\mathrm{H}$ & $\mathrm{H}$ & $\mathrm{H}$ \\
$\mathbf{2} \mathbf{b}$ & $\mathrm{OH}$ & $\mathrm{H}$ & $\mathrm{H}$ & $\mathrm{H}$ \\
$\mathbf{2}$ & $\mathrm{H}$ & $\mathrm{OH}$ & $\mathrm{H}$ & $\mathrm{H}$ \\
$\mathbf{2} \mathbf{d}$ & $\mathrm{H}$ & $\mathrm{H}$ & $\mathrm{OH}$ & $\mathrm{H}$ \\
$\mathbf{2}$ & $\mathrm{H}$ & $\mathrm{H}$ & $\mathrm{OCH}$ & $\mathrm{H}$ \\
$\mathbf{2}$ & $\mathrm{H}$ & $\mathrm{H}$ & $\mathrm{Br}$ & $\mathrm{H}$ \\
$\mathbf{2}$ & $\mathrm{OH}$ & $\mathrm{H}$ & $\mathrm{Br}$ & $\mathrm{H}$ \\
$\mathbf{2}$ & $\mathrm{OH}$ & $\mathrm{H}$ & $\mathrm{OH}$ & $\mathrm{H}$ \\
$\mathbf{2}$ & $\mathrm{OH}$ & $\mathrm{H}$ & $\mathrm{H}$ & $\mathrm{OH}$ \\
$\mathbf{2}$ & $\mathrm{H}$ & $\mathrm{OH}$ & $\mathrm{H}$ & $\mathrm{OH}$ \\
$\mathbf{2}$ & $\mathrm{H}$ & $\mathrm{OH}$ & $\mathrm{OH}$ & $\mathrm{H}$ \\
$\mathbf{2}$ & $\mathrm{H}$ & $\mathrm{OCH}$ & $\mathrm{OH}$ & $\mathrm{H}$ \\
$\mathbf{2}$ & $\mathrm{H}$ & $\mathrm{OH}$ & $\mathrm{OCH}$ & $\mathrm{H}$ \\
$\mathbf{2}$ & $\mathrm{OCH}$ & $\mathrm{H}$ & $\mathrm{H}$ & $\mathrm{OCH}_{3}$ \\
$\mathbf{2}$ & $\mathrm{OH}$ & $\mathrm{OH}$ & $\mathrm{OH}$ & $\mathrm{H}$ \\
$\mathbf{2}$ & $\mathrm{H}$ & $\mathrm{OH}$ & $\mathrm{OH}$ & $\mathrm{OH}_{3}$ \\
$\mathbf{2}$ & $\mathrm{H}$ & $\mathrm{OCH}$ & $\mathrm{OCH}$ & $\mathrm{OCH}_{3}$ \\
\hline
\end{tabular}

Table 2. Inhibitory Effects on Mushroom Tyrosinase of Phenylmethylenethiosemicarbazones $\mathbf{2} \mathbf{a}-\mathbf{q}$ and Its Analogue $\mathbf{2 r}$ as Compared with the Reference Inhibitors

\begin{tabular}{|c|c|c|c|}
\hline Compound & $\left.\mathrm{IC}_{50}(\mu \mathrm{mol} / 1)^{a}\right)$ & Compound & $\mathrm{IC}_{50}(\mu \mathrm{mol} / 1)^{a)}$ \\
\hline $2 \mathbf{a}$ & 1.93 & 2l & 5.68 \\
\hline $2 b$ & 0.38 & $2 \mathrm{~m}$ & 4.88 \\
\hline $2 c$ & 3.90 & $2 n$ & 87.5 \\
\hline $2 d$ & 0.41 & 20 & $>200$ \\
\hline $2 \mathrm{e}$ & 1.48 & $2 p$ & 115.2 \\
\hline $2 f$ & 0.28 & $2 q$ & 145.5 \\
\hline $2 g$ & 0.33 & $2 \mathbf{r}$ & 0.45 \\
\hline $2 \mathrm{~h}$ & 0.18 & Thiosemicarbazide $^{b}$ ) & $>2000$ \\
\hline $2 \mathbf{i}$ & 6.50 & 4-Hydroxybenzaldehyde ${ }^{c)}$ & 1220 \\
\hline $2 \mathrm{j}$ & 33.6 & 4-Methoxycinnamic acid $\left.{ }^{d}\right)$ & 410 \\
\hline $2 \mathbf{k}$ & 42.2 & Tropolone $e^{e}$ & 0.42 \\
\hline
\end{tabular}

a) Values were determined from logarithmic concentration-inhibition curves and are given as means of three experiments. b) The concentration of $2.00(\mathrm{~mm})$ corresponding to inhibition percentage, determined in this work, is $39.9 \%$. c) The reported $\mathrm{IC}_{5}$ values of $\mathrm{C}$ is $1.2 \mathrm{~mm}^{8}{ }^{8} \quad d$ ) The reported $\mathrm{IC}_{50}$ values of $\mathrm{B}$ is $0.42 \mathrm{~mm}^{25)} \quad$ e) Values in the literature is $0.40 \mu \mathrm{M}^{26)}$

$\mu \mathrm{M})$ presented at position-4 was replaced with methoxy group as in compound $\mathbf{2 e}\left(\mathrm{IC}_{50}=1.48 \mu \mathrm{M}\right)$, a decrease in inhibition potency was observed, while replacement of hydroxyl group at position-4 of compound $\mathbf{2 d}$ with a bromine subtituent as in compound $\mathbf{2} \mathbf{f}\left(\mathrm{IC}_{50}=0.28 \mu \mathrm{M}\right)$ led to a slight 
increase in inhibition activity. These results further confirmed that halogen atom as an electron-donating and hydrophobic group could strengthen the inhibitory effect on tyrosinase ${ }^{28)}$ while the methoxy group was not well tolerated.

Interestingly, incorporation of additional hydroxyl groups on the phenyl in the position- 4 of compound $\mathbf{2} \mathbf{b}$ afforded the most potent compound $\mathbf{2 h}\left(\mathrm{IC}_{50}=0.18 \mu \mathrm{M}\right)$, while its congeners $2 \mathbf{i}\left(\mathrm{IC}_{50}=6.50 \mu \mathrm{M}\right) \mathbf{2} \mathbf{j}\left(\mathrm{IC}_{50}=33.6 \mu \mathrm{M}\right)$ and $\mathbf{2} \mathbf{k}\left(\mathrm{IC}_{50}=\right.$ $42.2 \mu \mathrm{M})$ displayed a dramatic decline in inhibition activity. Compared with compound $\mathbf{2 h}$, compound $\mathbf{2 g}\left(\mathrm{IC}_{50}=0.33 \mu \mathrm{M}\right)$ bearing 2-hydroxyl group and 4-bromine substituent, respectively, exhibited a slight decline in activity. Unfortunately, replacement of two hydroxyl groups of compound $2 \mathbf{i}$ with two methoxy substituents to afford compound $2 \mathbf{n}\left(\mathrm{IC}_{50}=87.5 \mu \mathrm{M}\right)$ resulted in a loss of inhibition activity. To further investigate the effect of the number and position of hydroxyl substituents on phenyl ring, 2,3,4-trihydroxylated compound 2o, 3,4,5-trihydroxylated compound $\mathbf{2 p}$ and 3,4,5-trimethoxylated compound $\mathbf{2 q}$ were examined for the tyrosinase inhibitory potency. As shown in Table 2, compound 20 $\left(\mathrm{IC}_{50}>200 \mu \mathrm{M}\right)$ exhibited no inhibitory effect at the concentration of $200 \mu \mathrm{M}$, and compound $\mathbf{2 p}\left(\mathrm{IC}_{50}=115.2 \mu \mathrm{M}\right)$ and 2q $\left(\mathrm{IC}_{50}=145.5 \mu \mathrm{M}\right)$ displayed weaker inhibition activities. These results further confirmed that 2- and 4-hydroxy groups contributed to their inhibitory effect, while 3- and 5-hydroxyl groups and methoxy substituent were detrimental to their inhibition activities.

In addition, disubstituted compounds $\mathbf{2 h}, \mathbf{2 i}, \mathbf{2 j}, \mathbf{2} \mathbf{k}, \mathbf{2} \mathbf{l}$ and $\mathbf{2 m}$ exhibited more potent tyrosinase inhibition potencies than trisubstituted compounds $\mathbf{2 o}, \mathbf{2} \mathbf{p}$ and $\mathbf{2 q}$ indicating that the third substituent might hinder the correct docking of the inhibitor to the active site of tyrosinase. Moreover, replacement of benzene moiety of compound $2 \mathbf{a}\left(\mathrm{IC}_{50}=1.93 \mu \mathrm{M}\right)$ with furan group as in compound $2 \mathbf{r}\left(\mathrm{IC}_{50}=0.45 \mu \mathrm{M}\right)$ increased inhibition potency, suggesting that the electron-rich aromatic ring might be more favorable.

In order to further investigate the inhibition action of phenylmethylenethiosemicarbazones, experiments using UV/ vis spectroscopy were performed in which the enzyme was incubated with these inhibitors bearing a catechol moiety $(\mathbf{2} \mathbf{k}, \mathbf{0}, \mathbf{p})$ at various times. The results obtained showed that the change in absorbance at $475 \mathrm{~nm}$ had not been detected, suggesting that these compounds themselves cannot be oxidized by the enzyme as substrates. In addition, it should be noted that great improvement of tyrosinase inhibitory activity was obtained by coupling the corresponding benzaldehydes with thiosemicarbazide. These results indicated that the inhibition activity of these compounds was caused by the compounds themselves and not by their degradation products. ${ }^{17}$

DPPH Radical Scavenging Activity The melanin biosynthesis and food browning process also involved in non-enzymatic oxidation, and this oxidation process can be protected by radical scavengers. Therefore, in the present study, DPPH radical scavenging activities $\left(\mathrm{EC}_{50}\right)$ of the potent tyrosinase inhibitors $\left(\mathrm{IC}_{50}<10 \mu \mathrm{M}\right)$ were also evaluated by using the slightly modified method. ${ }^{24)}$ The results were summarized in Table 3. As shown in Table 3, all the select compounds exhibited potent DPPH radical scavenging activity with $\mathrm{EC}_{50}$ values ranged from 1.9 to $38.6 \mu \mathrm{M}$. Particularly, compounds $\mathbf{2 d}, \mathbf{2 e}, \mathbf{2 h}, \mathbf{2} \mathbf{i}$ and $\mathbf{2 l}$ exhibited more potent $\mathrm{DPPH}$ radical scavenging activity than Vc and TBHQ, which
Table 3. The $\mathrm{EC}_{50}$ Value of DPPH Radical Scavenging Activity of Compounds $\mathbf{2} \mathbf{a}-\mathbf{i}, \mathbf{2} \mathbf{l}-\mathbf{m}$ and $\mathbf{2 r}$

\begin{tabular}{cccc}
\hline \hline Compound & $\mathrm{EC}_{50}(\mu \mathrm{mol} / \mathrm{l})^{a)}$ & Compound & $\mathrm{EC}_{50}(\mu \mathrm{mol} / 1)^{a)}$ \\
\hline $\mathbf{2 a}$ & 53.2 & $\mathbf{2 h}$ & 5.52 \\
$\mathbf{2 b}$ & 10.8 & $\mathbf{2 i}$ & 2.62 \\
$\mathbf{2 c}$ & 38.6 & $\mathbf{2}$ & 4.20 \\
$\mathbf{2 d}$ & 5.42 & $\mathbf{2} \mathbf{m}$ & 11.4 \\
$\mathbf{2}$ & 6.67 & $\mathbf{2 r}$ & 7.81 \\
$\mathbf{2 f}$ & 10.5 & $\mathrm{Vc}^{b)}$ & 13.9 \\
$\mathbf{2 g}$ & 16.3 & $\mathrm{TBHQ}^{b)}$ & 7.40 \\
\hline
\end{tabular}

a) Values were determined from logarithmic concentration-scavenging curves and are given as means of three experiments. b) Ascorbic acid (Vc) and tertiary butyl hydroquinone (TBHQ) were used as the reference drug.
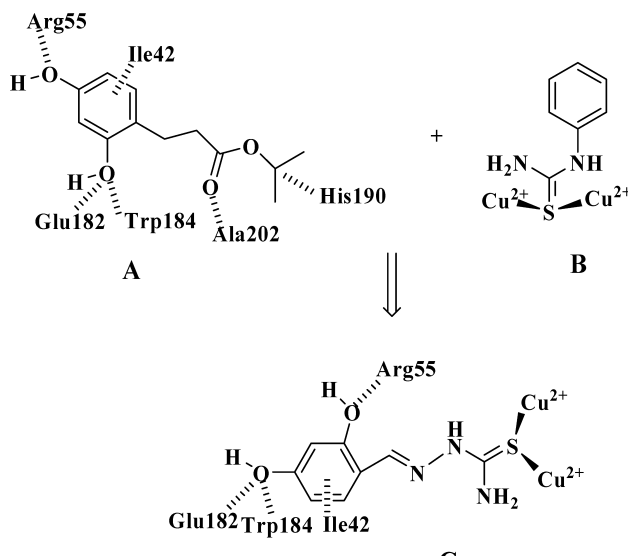

C

Fig. 2. Compound $\mathbf{2 h}$ Interaction with Mushroom Tyrosinase Three-Dimensional Active Site

(A) DPPA isopropyl ester interaction with the three-dimensional tyrosinase active site. $^{a}$ (B) Phenylthiourea chelating the binuclear copper of tyrosinase. ${ }^{a}$ (C) 2-[(2,4-Dihydroxyphenyl)methylene]thiosemicarbazone (compound $\mathbf{2 h}$ ) interaction with tyrosinase active site. ${ }^{\mathrm{b}}$ The sulfur atoms of thiosemicarbazide moiety chelate the binuclear copper active site, while the two hydroxyl groups of 2,4-resorcinol moiety form three hydrogen bonds at the active site of tyrosinase, two with the nitrogen atoms of the amino acids Arg55 and Trp184 on the side chains and one with the carboxylic group of the Glu182. ${ }^{a} \mathrm{~A}$ and B represented the previous reported models. ${ }^{b} \mathrm{C}$ represented our proposed model.

are commonly used in food industry as antioxidants. ${ }^{29)}$

Inhibitory Mechanism Analysis The crystallographic structure of tyrosinase has been established, ${ }^{30,31)}$ enabling a close look at its three-dimensional structure and a better understanding of its mechanism of action. Within the structure, there are two copper ions in the active center of tyrosinase and it was deduced that there is a lipophilic long-narrow gorge near to the active center, and further investigation showed that the hydrophobic domain is composed of some bioactive amino acids, such as Arg 55, Trp 184, Glu 182, Ile 42, His 190 and Ala 202. ${ }^{32)}$

On the basis of the above-mentioned structure, a number of polyphenol derivatives and especially such compounds containing a resorcinol subunit have been extensively investigated. ${ }^{1,11,31)}$ Khatib et al. designed a series of 3-(2,4-dihydroxyphenyl)-propionic acid (DPPA) isopropyl esters as tyrosinase inhibitors, and the molecular docking study indicated that the most active DPPA isopropyl ester could form four hydrogen bonds and two hydrophobic interactions with lipophilic gorge, close to the copper ion couple (the reported interaction model shown in Fig. 2A). ${ }^{11)}$ In addition, the sulfur atom of thioureas moiety exhibited strong affinity for the binuclear copper active site, and the reported interaction model 
was illustrated in Fig. 2B. ${ }^{31,33)}$

In this investigation, compound $\mathbf{2 h}$ bearing 2,4-resorcinol and thiosemicarbazide moieties was found to be the most potent tyrosinase inhibitor. According to the models shown in Fig. 2A and Fig. 2B, the interaction model (Fig. 2C) between mushroom tyrosinase and compound $\mathbf{2} \mathbf{h}$ was proposed. Figure $2 \mathrm{C}$ demonstrated that the thiosemicarbazide moiety could fit the binuclear copper active site well because the sulfur atom formed strong chelation with the binuclear copper of tyrosinase. Such interaction acted like a bridge to link the hydroxyl group and the hydrophobic protein pocket surrounding the binuclear copper active site, which facilitated the hydroxyl group and the active site of enzyme to interact. Thus, the two hydroxyl groups of 2,4-resorcinol moiety formed three hydrogen bonds at the active site of tyrosinase, close to the copper ion couple, two with the nitrogen atoms of the amino acids Arg55 and Trp184 on the side chains and one with the carboxylic group of the Glu182. Furthermore, a hydrophobic interaction of compound $\mathbf{2 h}$ was also possible between its resorcinol aromatic ring and Ile 42. For the other compounds, due to the stereo-hindrance or bad position of hydroxyl group, it would make these compounds get close to the active center of tyrosinase harder, and even if these compounds could enter the active center to form the chelation with the binuclear copper, the weak interactions with the hydrophobic protein pocket of enzyme would make the chelation unstable.

Meanwhile, inspired by the model of tyrosinase three-dimensional structure, we hypothesized that the presence of a proper hydrophobic subunit connected to the resorcinol aromatic ring of compound $\mathbf{2 h}$, might interact with His 190 and Ala 202 in the hydrophobic protein pocket, leading to an improved tyrosinase inhibitory activity; such a compound will be considered for future synthesis.

\section{Conclusion}

In this study, a series of hydroxy- or methoxy-substituted phenylmethylenethiosemicarbazones were designed, synthesized and evaluated as mushroom tyrosinase inhibitors. The results demonstrated that most of compounds had potent tyrosinase inhibitory activities. Particularly, compounds 2b, $\mathbf{2 f}, \mathbf{2 g}$ and $\mathbf{2 h}$ exhibited more potent inhibitory effects than tropolone, one of the best tyrosinase inhibitors known so far. Preliminary structure-activity relationships analysis indicated that: (1) the thiosemicarbazide moiety was efficacious for the inhibitory activity; (2) the 2- and 4-hydroyl group on the phenyl ring was an essential requirement for tyrosinase inhibition; (3) the electron-rich aromatic ring contributed to their tyrosinase inhibition activity. In addition, DPPH radical scavenging activity of select compounds $\left(\mathrm{IC}_{50}<10 \mu \mathrm{M}\right)$ were also evaluated, and these compounds exhibited potent DPPH radical scavenging activity with $\mathrm{EC}_{50}$ values ranged from 1.9 to $38.6 \mu \mathrm{M}$. These results suggested that such compounds might be utilized for the development of new candidate for treatment of dermatological disorders, and further development of such compounds may be of interest.

Acknowledgment This work was supported by the Natural Science Foundation of Guangdong Province, China (2004B30101007).

\section{References and Notes}

1) Song K. K., Huang H., Han, P., Zhang C. L., Shi Y., Chen Q. X., Biochem. Biophys. Res. Commun., 342, 1147-1175 (2006).

2) Yokochi N., Morita T., Yagi T., J. Agric. Food Chem., 51, 2733-2736 (2003).

3) Nerya O., Musa R., Khatib S., Tamir S., Vaya J., Phytochemistry, 65, 1389-1395 (2004)

4) Maeda K., Fukuda M., J. Soc. Cosmet. Chem., 42, 361-363 (1991).

5) Maeda K., Fukuda M., J. Pharm. Exp. Ther, 276, 765-769 (1996).

6) Chen Q. X., Kubo I., J. Agric. Food Chem., 50, 4108 - 4112 (2002).

7) Shiino M., Watanabe Y., Umezawa K., Bioorg. Med. Chem., 9, 12331240 (2001).

8) Seo S. Y., Sharma V. K., Sharma N., J. Agric. Food Chem., 51, $2837-$ 2853 (2003).

9) Kubo I., Kinst-Hori I., Planta Med., 65, 19-22 (1999).

10) Okombi S., Rival D., Bonnet S., Mariotte A. M., Perrier E., Boumendjel A., J. Med. Chem., 49, 329-333 (2006).

11) Khatib S., Nerya O., Musa R., Tamir S., Pete T., Vaya J., J. Med. Chem., 50, 2676-2681 (2007).

12) Briganti S., Camera E., Picardo M., Pigment Cell. Res., 16, 101-110 (2003).

13) Khan K. M., Mughal U. R., Khan M. T. H., Perveen S., Ullah Z., Choudhary M. I., Bioorg. Med. Chem., 14, 344-351 (2006).

14) Um S. J., Park M. S., Park S. H., Han H. S., Kwon Y. J., Sin H. S., Bioorg. Med. Chem., 11, 5345-5352 (2003).

15) Nihei K. I., Yamagiwa Y., Kamikawa T., Kubo I., Bioorg. Med. Chem. Lett., 14, 681-683 (2004).

16) Jun N., Hong G., Jun K., Bioorg. Med. Chem., 15, 2396-2402 (2007).

17) Ley J. P., Bertram H. J., Bioorg. Med. Chem., 9, 1879-1885 (2001).

18) Miyazawa M., Oshima T., Koshio K., Itsuzaki Y., Anzai J., J. Agric. Food Chem., 51, 6953-6956 (2003).

19) Okombi S., Rival D., Bonnet S., Mariotte A. M., Perrier E., Boumendjel A., Bioorg. Med. Chem. Lett., 16, 2252-2255 (2006).

20) Khatib S., Nerya O., Musa R., Shmuel M., Tamir S., Vaya J., Bioorg. Med. Chem., 13, 433-441 (2005).

21) Liu J. B., Yi W., Wan Y. Q., Ma L., Song H. C., Bioorg. Med. Chem., 16, 1096-1102 (2008).

22) Liu J. B., Cao R. H., Yi W., Ma C. M., Wan Y. Q., Zhou B. H., Ma L., Song H. C., Eur. J. Med. Chem., 44, 1737-1778 (2009).

23) Hearing V. J. Jr., "Methods in Enzymology," Vol. 142, ed. by Kaufman S., Academic, New York, 1987, p. 155.

24) Iwai K., Kishimoto N., Kakino Y., Mochida K., Fujita T., J. Agric. Food Chem., 52, 4893-4898 (2004).

25) Shi Y., Chen Q. X., Wang Q., Song K. K., Qiu L., Food Chem., 92, 707-712 (2005).

26) Kahn V., Andrawis A., Phytochemistry, 24, 905-908 (1985).

27) Cho S. J., Roh J. S., Sun W. S., Kim S. H., Park K. D., Bioorg. Med. Chem. Lett., 16, 2682-2684 (2006).

28) Han P., Chen C. Q., Zhang C. L., Song K. K., Zhou H. T., Chen Q. X., Food Chem., 107, 797-803 (2008).

29) Yi W., Wu X. Q., Cao R. H., Song H. C., Ma L., Food Chem., 117, $381-386$ (2009).

30) Matoba Y., Kumagai T., Yamamoto A., Yoshitsu H., Sugiyama M., J. Biol. Chem., 281, 8981-8990 (2006).

31) Klabunde T., Eicken C., Nature Struct. Biol., 5, $1084-1090$ (1998).

32) Shimizu K., Kondo R., Sakai K., Planta Med., 66, 11-15 (2000).

33) Daniel J., U.S. Patent 2006135618 (2006). 